\title{
AN IGBT DRIVEN SLOTTED BEAM PIPE KICKER FOR SPEAR III INJECTION*
}

\author{
Chris Pappas, Richard Cassel, SLAC, Menlo Park, CA 95001
}

\begin{abstract}
The SPEAR III injection kicker system is composed of three kicker magnets, K1, K2, and K3. These magnets, along with the power modulators to drive them constitute an injection system which will be used to deflect an incoming electron beam with an energy of $3.3 \mathrm{GeV}$ by an angle of $2.5 \mathrm{mrad}$ for $\mathrm{K} 1$ and $\mathrm{K} 3$, and $1 \mathrm{mrad}$ for $\mathrm{K} 2$. The pulse shape of the magnetic field in the three magnets must be matched in order to preserve a closed orbit. The pulse duration is required to be less than $780 \mathrm{~ns}$, with rise and fall times of less than $375 \mathrm{~ns}$, and a pulse repetition frequency of $10 \mathrm{~Hz}$. The aperture of all three magnets is $60 \times 34 \mathrm{~mm}$ in an 8 inch vacuum vessel. The magnetic length is $1.2 \mathrm{~m}$ for $\mathrm{K} 1$ and $\mathrm{K} 3$, and $0.6 \mathrm{~m}$ for $\mathrm{K} 2$ [1]. The magnet design employs a slotted beam pipe which is shorted at one end. A solid state IGBT based, induction type of modulator drives the magnets. Modulators for K1 and $\mathrm{K} 3$ consist of eight $4.5 \mathrm{kV}, 600 \mathrm{~A}$ IGBTs, and eight Finemet magnet cores with four $22.5 \mathrm{Ohm}$ output cables to drive $2381 \mathrm{~A}$ into the magnets. The modulator for $\mathrm{K} 2$ uses four IGBTs and cores, and 8 output cables to produce a 2619 A pulse. Cables of length greater than one half the pulse width must be used in order to avoid reflections from the shorted magnet. The design charge voltage for the modulators is $20 \mathrm{kV}$ for $\mathrm{K} 1$ and $\mathrm{K} 3$. This paper describes the magnet and modulator design, as and presents test data from a prototype system.
\end{abstract}

\section{MAGNET DESIGN}

The SPEAR III kicker magnet design is based on a design developed at Delta [2]. The magnet is constructed by placing a slotted copper pipe inside a stainless steel vacuum vessel. Four slits are cut along the length of the pipe to create the two busses along with image current paths. Both busses are driven from the one end by a bipolar modulator, and are shorted at the opposite end. A diagram of the magnet is shown in Figure 1. A cross section of the magnet along with the flux lines when the magnet is filled as calculated by MAXWELL is shown in Figure 2. Figure 3 shows the magnetic field profile and calculated gain in a cross section of the magnet.

Both the busses and the image current returns are water cooled. The busses are made from copper to prevent field penetration into the conductors, which will enlarge the effective size of the slits and increase the gain of the magnet during the pulse if the pipe were made from stainless steel. Another consideration is the size of the vacuum vessel which affects both the gain of the magnet, and the magnet impedance and thus the field rise time since the magnet is driven by a modulator with a fixed output impedance. The two affects are in opposition, and a compromise was reached with the inside diameter of the vacuum vessel set to eight inches. This results in a calculated magnetic gain of $8.4 \mu \mathrm{T} / \mathrm{A}$, and an impedance of $76 \Omega$, or $478 \mathrm{nH} / \mathrm{m}$.

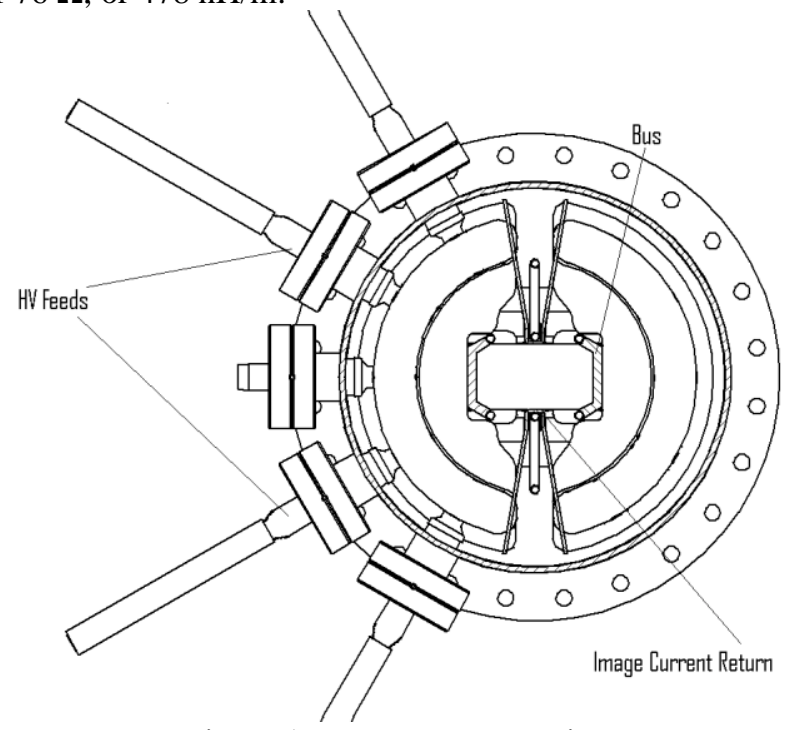

Figure 1. Magnet cross section.

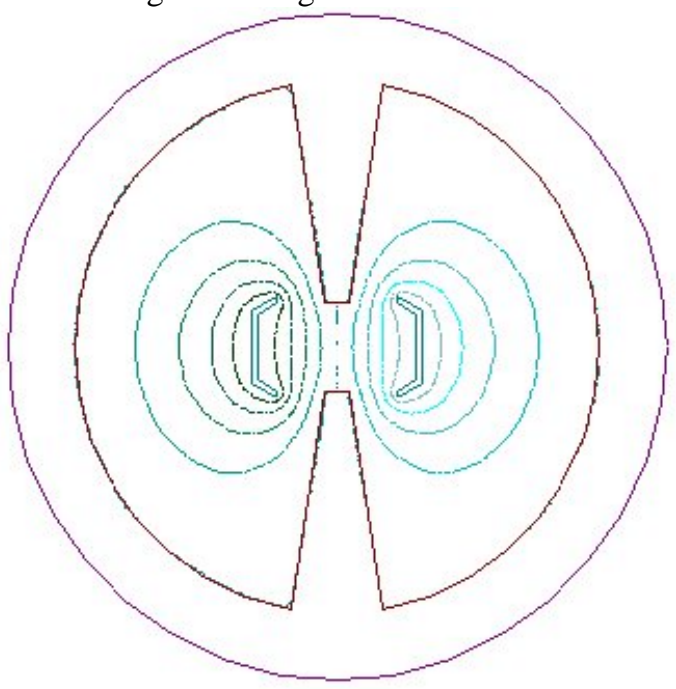

Figure 2. Cross section and flux lines in magnet.

Preliminary testing of the magnet has begun, and the gain of the magnet has been measured. Figure 4 shows the integrated $\mathbf{B}$ field, normalized to $1 \mathrm{~A}$, and $1 \mathrm{~m}$. Note the measured gain of approximately $8.7 \mu \mathrm{T} / \mathrm{A}$. Also note that the rise and fall times are somewhat slower than specified, which can be attributed to excessive leakage inductance of the transformer. 


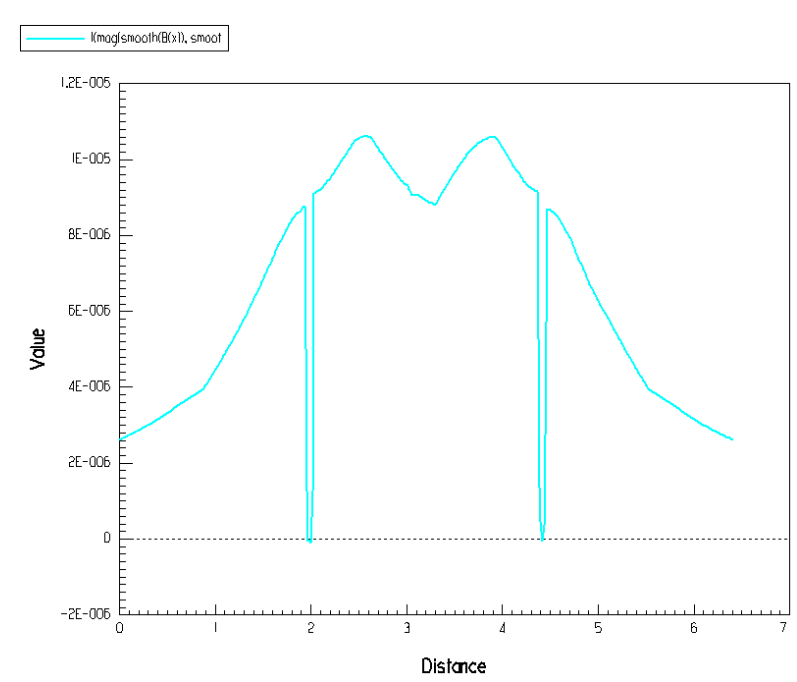

Figure3. MAXWELL calculated magnet gain.

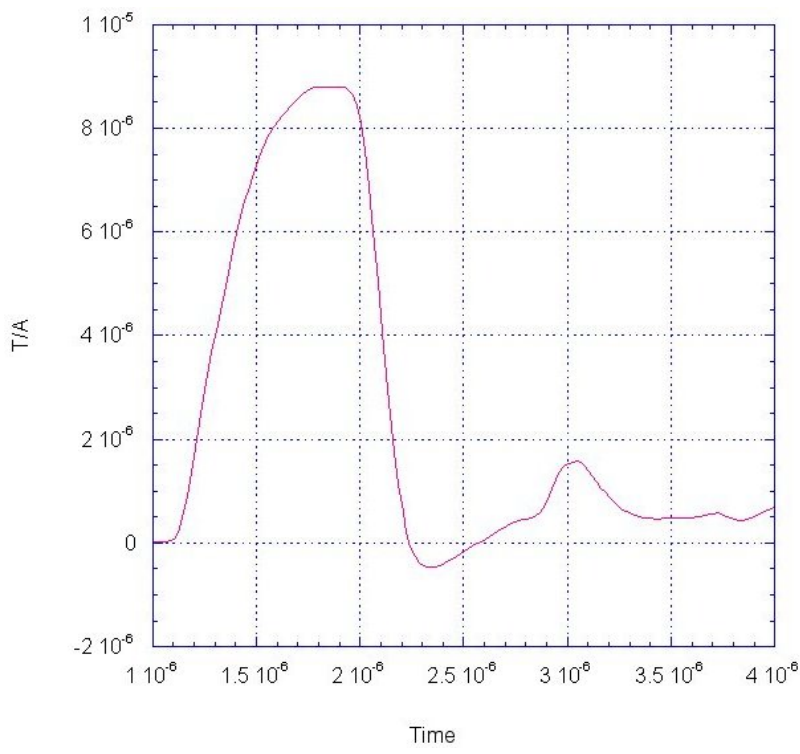

Figure 4. Normalized magnet gain.

\section{MODULATOR DESIGN}

The modulator to drive the SPEAR III kicker magnet is a solid state design which uses IGBTs to drive multiple primaries of a "fractional turn" transformer in parallel [3][4]. Each primary is a single turn, and the secondary is made by passing a single turn through a torroidal core. A schematic of the circuit is shown in Figure 5, and a photo of a four stack proto type is shown in Figure 6. The IGBTs used are Powerex CM600HB90H, 4500 V, $600 \mathrm{~A}$ modules. The core material used is Hitachi Metals, Finemet $3 \mathrm{H}$, non-magnetostrictive material, and the output cable is Times Microwave AA8481, $22.5 \Omega$ coax. Circuit operation is as follows. The IGBT closes, producing a pulse which travels down the output cable and into the magnet.

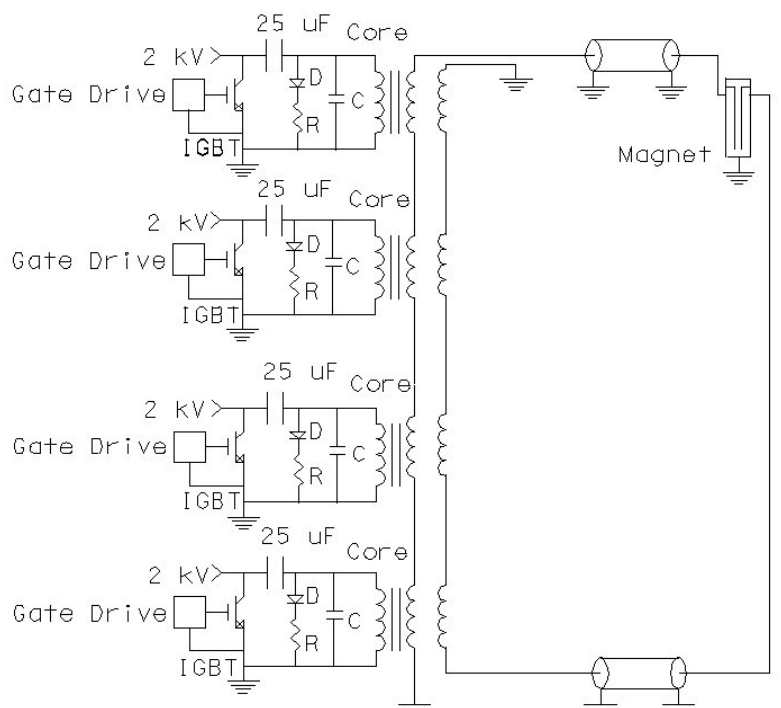

Figure 5. Schematic of K2 modulator.

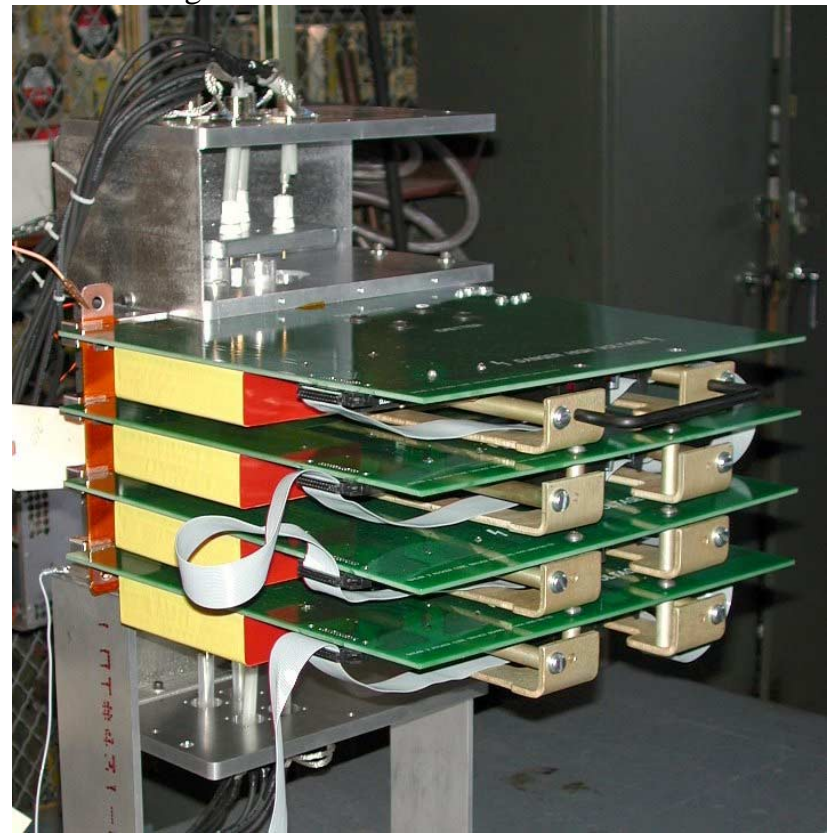

Figure 6. Photo of four stack proto type modulator.

The shorted magnet doubles the current upon reflection. Before the reflected wave reaches the modulator, the switch opens, and the reflected pulse is terminated by the matching resistor and diode network. Bi-polar outputs are achieved by passing the single turn secondaries through the core in opposite directions. Since the K2 magnet is one half the length, and therefore one half the inductance as $\mathrm{K} 1$ and $\mathrm{K} 3$, the output impedance of the $\mathrm{K} 2$ modulator was reduced by a factor of two by using four output cables per buss instead of the two used in the K1, K3 modulators. A SPICE simulated magnet current pulse for the $\mathrm{K} 2$ magnet is shown in Figure 7.

Testing is progressing on the modulator at SLAC, while the magnet has been shipped to Lawrence Berkeley Laboratories for beam impedance measurements. The original transformer secondaries design used four pipes, each of 1 inch diameter, passing through the core stack. 


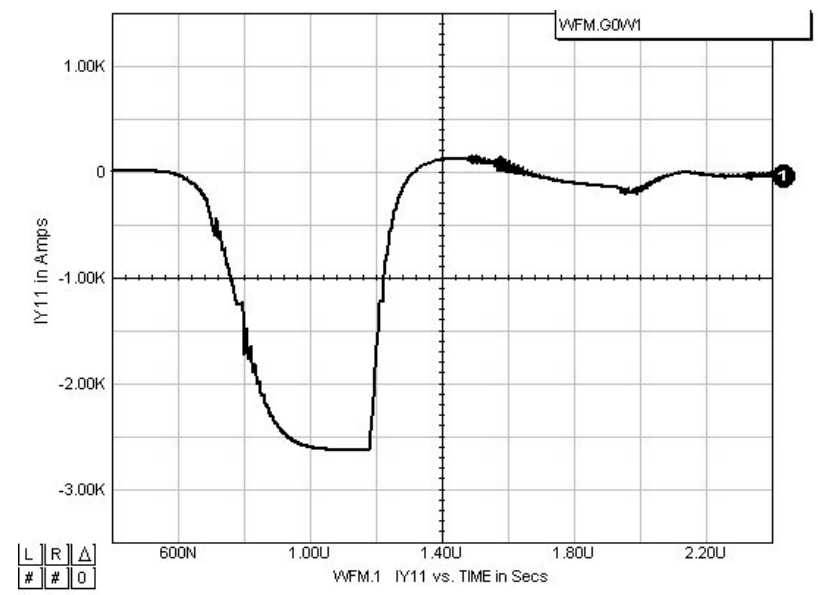

Figure 7. SPICE simulation of K2 current.

This design is very simple and inexpensive mechanically, but has a leakage inductance of approximately $400 \mathrm{nH} / \mathrm{m}$. This inductance, along with the inductance of the high voltage connections is excessive. A current pulse and voltage waveform across the core with this design, and a shorted load, are shown in Figure 8.

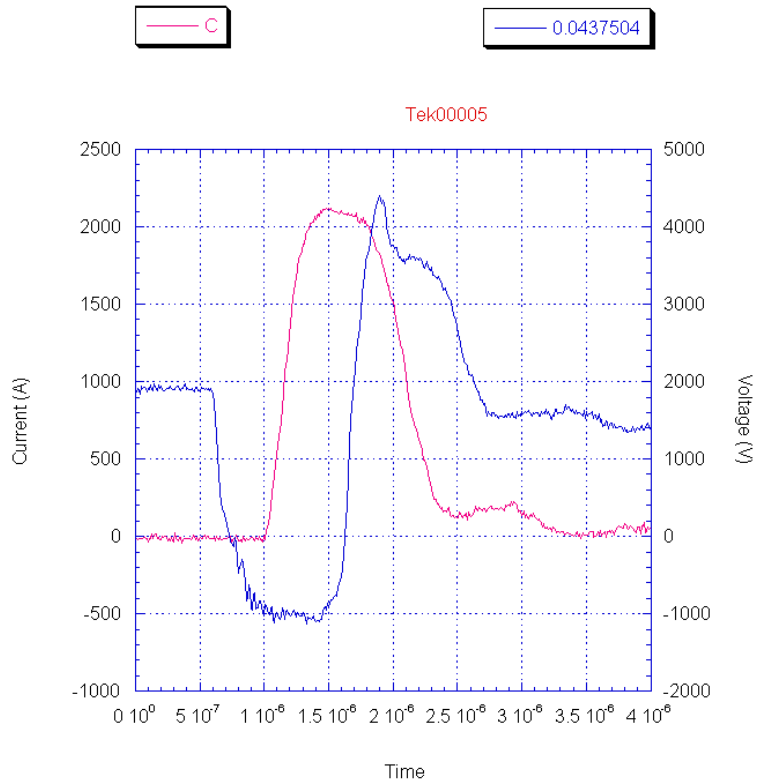

Figure 8. Current pulse and core voltage with four pipe secondary design.

Clearly the rise and fall times can be improved by a reduction in the transformer leakage inductance, which can be achieved by filling more of the transformer inner dimension area. A new secondary design is now planned, that uses a single 3.35 inch aluminum rod which is split down the middle and connected from opposing ends to create the two secondary windings. A cross section of the geometry is shown in Figure 9, and MAXWELL calculations show that the leakage inductance of this configuration is less than $60 \mathrm{nH} / \mathrm{m}$. The high voltage connections should also have less inductance than the old design. Figure 8 also shows a voltage spike on the IGBT caused by the forward recovery of the diode. This spike will exceed the voltage rating of the IGBT for operation of $\mathrm{K} 2$, and must be limited either by a snubber across the diode, or by using another diode with lower forward recovery.

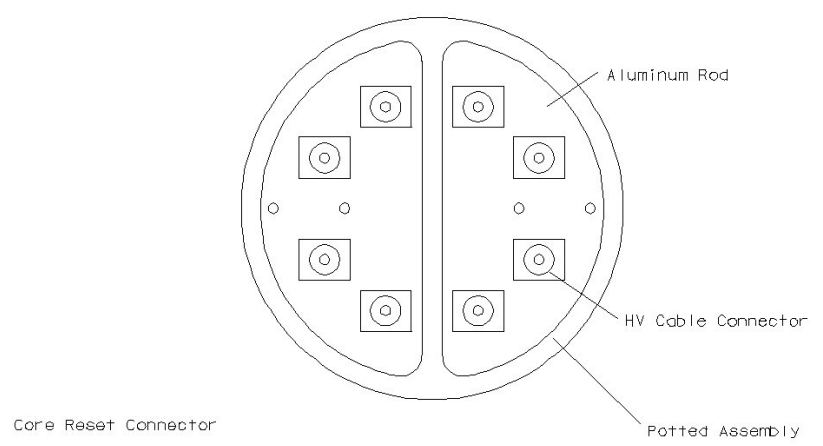

Figure 9. View of potted, split rod secondary assembly.

\section{CONCLUSIONS}

The slotted beam pipe kicker magnet has performed almost exactly as designed, with the only question remaining to be answered is that of its beam impedance. A few problems have been encountered with the solid state induction modulator design, the most serious of which is the high leakage inductance of the original secondary geometry. This problem should be corrected by the split rod design above.

\section{ACKNOWLEDGEMENTS}

The authors would like to express there thanks to J. Langton and D. Arnett for help with the mechanical design of the magnet, and D. Arnett and W. Gorecki for their help with the mechanical design of the modulator. Thanks to W. Gorecki and J. Olszewski for PCB design, and J. Krzaszczak, P. Blum, and D. Moreno for help with proto typing, assembly, and testing.

\section{REFERENCES}

[1] Sebek, J.,SPEAR3 Injection Kicker System,PAC2001.

[2] Blokesch, G., A Slotted-pipe Kicker for High-current Storage Rings, Nuclear Instruments and Methods in Physics Research, A 388 (1994).

[3] Pappas, C., Damping Ring Kickers for the Next Linear Collider, PAC 1999, p1500.

[4] Cassel, R.L., A Solid State Induction Modulator for SLAC NLC, PAC 1999, p 1494. 